\title{
Relations Between Seepage Velocities in Immiscible, Incompressible Two-Phase Flow in Porous Media
}

\author{
Alex Hansen $^{1}$ (D) Santanu Sinha ${ }^{2}$ - Dick Bedeaux ${ }^{3}$. Signe Kjelstrup ${ }^{3}$. \\ Magnus Aa. Gjennestad ${ }^{1} \cdot$ Morten Vassvik $^{1}$
}

Received: 17 December 2017 / Accepted: 3 September 2018 / Published online: 10 September 2018

(c) The Author(s) 2018

\begin{abstract}
Based on thermodynamic considerations, we derive a set of equations relating the seepage velocities of the fluid components in immiscible and incompressible two-phase flow in porous media. They necessitate the introduction of a new velocity function, the co-moving velocity. This velocity function is a characteristic of the porous medium. Together with a constitutive relation between the velocities and the driving forces, such as the pressure gradient, these equations form a closed set. We solve four versions of the capillary tube model analytically using this theory. We test the theory numerically on a network model.
\end{abstract}

Keywords Immiscible two-phase flow $\cdot$ Thermodynamics $\cdot$ Seepage velocity $\cdot$ Capillary tube model $\cdot$ Network models

\section{Introduction}

The simultaneous flow of immiscible fluids through porous media has been studied for a long time (Bear 1988). It is a problem that lies at the heart of many important geophysical and industrial processes. Often, the length scales in the problem span numerous decades, from the pores measured in micrometers to reservoir scales measured in kilometers. At the largest

\footnotetext{
$凶$ Alex Hansen

Alex.Hansen@ntnu.no

Santanu Sinha

santanu@csrc.ac.cn

Dick Bedeaux

Dick.Bedeaux@ntnu.no

Signe Kjelstrup

Signe.Kjelstrup@ntnu.no

1 PoreLab and Department of Physics, Norwegian University of Science and Technology, 7491 Trondheim, Norway

2 CSRC, 10 East Xibeiwang Road, Haidian District, Beijing 100193, China

3 PoreLab and Department of Chemistry, Norwegian University of Science and Technology, 7491 Trondheim, Norway
} 
scales, the porous medium is treated as a continuum governed by effective equations that encode the physics at the pore scale.

The problem of tying the pore scale physics together with the effective description at large scale is the upscaling problem. In 1936, Wycoff and Botset proposed a generalization of the Darcy equation to immiscible two-phase flow (Wyckoff and Botset 1936). It is instructive to reread Wycoff and Botset's article. This is where the concept of relative permeability is introduced. The paper is eighty years old, and yet it is still remarkably modern. Capillary pressure was first considered by Richards as early as 1931 (Richards 1931). In 1940, Leverett combined capillary pressure with the concept of relative permeability, and the framework which dominates all later practical analyses of immiscible multiphase flow in porous media was in place (Leverett 1940).

However, other theories exist Larson et al. (1981), Gray and Hassanizadeh (1989), Hassanizadeh and Gray (1990), Hassanizadeh and Gray (1993a), Hassanizadeh and Gray (1993b), Hilfer (1998), Hilfer and Besserer (2000), Gray and Miller (2005), Hilfer (2006a, b, c), Hilfer and Döster (2010), Niessner et al. (2011), Döster et al. (2012), Bentsen and Trivedi (2013), Hilfer et al. (2015), Hassanizadeh (2015) and Ghanbarian et al. (2016). These theories are typically based on a number of detailed assumptions concerning the porous medium and concerning the physics involved.

It is the aim of this paper to present a new theory for flow in porous media that is based on thermodynamic considerations. In the same way as Buckley and Leverett's analysis based on the conservation of the mass of the fluids in the porous medium led to their Buckley-Leverett equation (Buckley and Leverett 1942), the thermodynamical considerations presented here lead to a set of equations that are general in that they transcend detailed assumptions about the flow. This is in contrast to the relative permeability equations that rely on a number of specific physical assumptions.

The theory we present focuses on one aspect of thermodynamic theory. We see it as a starting point for a more general analysis based on non-equilibrium thermodynamics (Kondepudi and Prigogine 1998; Kjelstrup and Bedeaux 2008; Kjelstrup et al. 2017) which combines conservation laws with the laws of thermodynamics. The structure of the theory, already as it is presented here, is reminiscent of the structure of thermodynamics itself: We have a number of variables that are related through general thermodynamic principles leaving an equation of state to account for the detailed physics of the problem. To our knowledge, the analysis we present here has no predecessor. However, the framework in which it resides is that originally laid out by Gray and Hassanizadeh (1989), Hassanizadeh and Gray (1990), Hassanizadeh and Gray (1993a), Hassanizadeh and Gray (1993b) and Gray and Miller (2005). This approach, named Thermodynamically Constrained Averaging Theory (TCAT), has generated a large body of work since its inception; see Gray and Miller (2014).

The theory we present concerns relations between the flow rates, or equivalently, the seepage velocities of the immiscible fluids. We do not discuss relations between the seepage velocities and the driving forces that create them, such as a pressure gradient. In this sense, we are presenting a "kinetic" theory of immiscible two-phase flow in porous media, leaving out the "dynamic" aspects which will be addressed elsewhere. Our main result is a set of equations between the seepage velocities that together with a constitutive relation between the average seepage velocity and the driving forces lead to a closed set of equations.

We consider only one-dimensional flow in this paper, deferring to later the generalization to three dimensions. The fluids are assumed to be incompressible.

In Sect. 2, we describe the porous medium system we consider. We review the key concepts that will be used in the subsequent discussion. In particular, we discuss the relation between average seepage velocity and the seepage velocities of each of the two fluids. 
In Sect. 3, we introduce our thermodynamic considerations. We focus on the observation that the total volumetric flow rate is an Euler homogeneous function of the first order. This allows us to define two thermodynamic velocities as derivatives of the total volumetric flow. We then go on in Sect. 4 to deriving several equations between the thermodynamic velocities, one of which is closely related to the Gibbs-Duhem equation in thermodynamics. In Sect. 4.1, we point out that the seepage velocity of each fluid is generally not equal to the corresponding thermodynamic velocity. This is due to the constraints that the geometry of the porous medium puts on how the immiscible fluids arrange themselves. In ordinary thermodynamics, such constraints are not present and questions of this type do not arise. We relate the thermodynamic and seepage velocities through the introduction of a co-moving velocity function. This comoving velocity is a characteristic of the porous medium and depends on the driving forces only through the velocities and the saturation.

We discuss in Sect. 5 what happens when the thermodynamic and seepage velocities coincide, when the wetting and non-wetting seepage velocities coincide and when the thermodynamic wetting and non-wetting velocities coincide. Normally, the coincidences appear at different saturations. However, under certain conditions, the three coincide. When this happens, the fluids behave as if they were miscible.

In Sect. 6, we write down the full set of equations to describe immiscible two-phase flow in porous media. These equations are valid on a large scale where the porous medium may be seen as a continuum.

In Sect. 7, we analyze four versions of the capillary tube model (Scheidegger 1953, 1974) within the concept of the representative elementary volume (REV) developed in the previous sections. This allows us to demonstrate these concepts in detail and to demonstrate the internal consistency of the theory.

In Sect. 8, we analyze a network model (Aker et al. 1998) for immiscible two-phase flow within the framework of our theory. We calculate the co-moving velocity for the model using a square and a hexagonal lattice. The co-moving velocity is to within the level of the statistical fluctuations equal for the two lattice topologies. We compare successfully the measured seepage velocities for each fluid component with those calculated from our theory. Lastly, we use our theory to predict the coincidence of the three saturations defined in Sect. 5 and verify this prediction numerically.

Section 9 summarizes the results from the previous sections together with some further remarks on the difference between our approach and that of relative permeability. We may nevertheless anticipate here what will be our conclusions. Through our theory, we have accomplished two things. The first one is to construct a closed set of equations for the seepage velocities as a function of saturation based solely on thermodynamic principles. We do not propose relations between seepage velocities and driving forces such as pressure gradient here. Our discussion is solely based on relations between the seepage velocities. The second accomplishment is to pave the way for further thermodynamic analysis by identifying the proper thermodynamic variables that relate to the flow rates.

\section{Defining the System}

The aim of this paper is to derive a set of equations on the continuum level where differentiation makes sense. We define a representative elementary volume- $\mathrm{REV}$ - as a block of porous material with no internal structure filled with two immiscible and incompressible fluids: It is described by a small set of parameters which we will now proceed to define. 


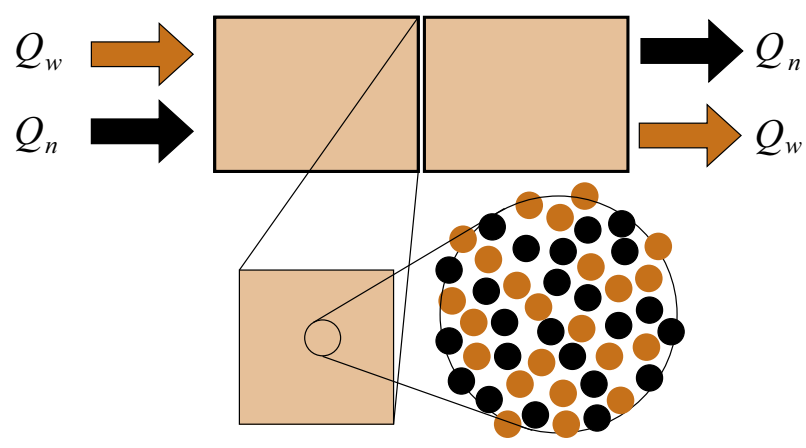

Fig. 1 In the upper part of the figure, we see the REV from the side. There is a flow $Q=Q_{w}+Q_{n}$ across it. An imaginary cut is made through the REV in the direction orthogonal to the flow. In the lower left corner, the surface of the imaginary cut is illustrated. A magnification of the surface of the cut is shown in the lower right corner. The pore structure is illustrated as brown and black circles. The pores that are brown are filled with wetting fluid, and the pores that are black are filled with non-wetting fluid. The wetting fluid-filled pores form in total an area $A_{w}$, and the non-wetting fluid-filled pores form in total an area $A_{n}$. The total pore area of the imaginary cut in the lower left corner is $A_{p}=A_{w}+A_{n}=A \phi$

We illustrate the REV in Fig. 1. It is a block of homogeneous porous material of length $L$ and area $A$. We seal off the surfaces that are parallel to the $L$-direction (the flow direction). The two remaining surfaces, each with area $A$, are kept open and act as inlet and outlet for the fluids that are injected and extracted from the REV. The porosity is

$$
\phi \equiv \frac{V_{p}}{V}=\frac{V_{p}}{A L},
$$

where $V_{p}$ is the pore volume and $V=A L$ the total volume of the REV. Due to the homogeneity of the porous medium, any cross section orthogonal to the axis along the $L$-direction (named the $x$ axis for later) will reveal a pore area that fluctuates around the value

$$
A_{p}=\frac{V_{p}}{L}=\phi A
$$

while the solid matrix area fluctuates around

$$
A_{s} \equiv A-A_{p}=(1-\phi) A .
$$

The homogeneity assumption consists in the fluctuations being so small that they can be ignored.

There is a time-averaged volumetric flow rate $Q$ through the REV (see Fig. 1). The volumetric flow rate consists of two components, $Q_{w}$ and $Q_{n}$, which are the volumetric flow rates of the more wetting ( $w$ for "wetting") and the less wetting ( $n$ for "non-wetting") fluids with respect to the porous medium. We have

$$
Q=Q_{w}+Q_{n}
$$

All flows have as frame of reference the non-deformable solid matrix.

In the porous medium, there is a volume $V_{w}$ of incompressible wetting fluid and a volume $V_{n}$ of incompressible non-wetting fluid so that $V_{p}=V_{w}+V_{n}$. We define the wetting and non-wetting saturations $S_{w}=V_{w} / V_{p}$ and $S_{n}=V_{n} / V_{p}$ so that

$$
S_{w}+S_{n}=1 \text {. }
$$


We define the wetting and non-wetting pore areas $A_{w}$ and $A_{n}$ as the parts of the pore area $A_{p}$ which are filled with the wetting or the non-wetting liquids, respectively. As the porous medium is homogeneous, we will find the same averages $A_{w}$ and $A_{n}$ in any cross section through the porous medium orthogonal to the flow direction. This is illustrated in Fig. 1. We have that $A_{w} / A_{p}=\left(A_{w} L\right) /\left(A_{p} L\right)=V_{w} / V_{p}=S_{w}$ so that

$$
A_{w}=S_{w} A_{p} \text {. }
$$

Likewise,

$$
A_{n}=S_{n} A_{p}=\left(1-S_{w}\right) A_{p}
$$

where

$$
A_{p}=A_{w}+A_{n} .
$$

We define the seepage velocities for the two immiscible fluids, $v_{w}$ and $v_{n}$ as

$$
v_{w}=\frac{Q_{w}}{A_{w}},
$$

and

$$
v_{n}=\frac{Q_{n}}{A_{n}} .
$$

Hence, Eq. (4) may be written

$$
Q=A_{w} v_{w}+A_{n} v_{n} .
$$

We finally define a seepage velocity associated with the total flow rate $Q$ as

$$
v=\frac{Q}{A_{p}} .
$$

By using Eqs. (6)-(8) and (9), (10) and (12), we transform (11) into

$$
v=S_{w} v_{w}+S_{n} v_{n} .
$$

All the variables in this equation can be measured experimentally.

The extensive variables describing the REV, among them the wetting and non-wetting pore areas $A_{w}$ and $A_{n}$ and the wetting and non-wetting volumetric flow rates $Q_{w}$ and $Q_{n}$, are averages over the REV, and their corresponding intensive variables $S_{w}, S_{n}, v_{w}$ and $v_{n}$ are therefore by definition a property of the REV.

This definition of a REV is the same as that used by Gray and Miller (2005) in their thermodynamically constrained averaging theory and in the earlier literature on thermodynamics of multiphase flow, see, for example, Hassanizadeh and Gray (1990). The REV can be regarded as homogeneous only on the REV scale. With one driving force, the variables on this scale can be obtained, knowing the microscale ensemble distribution (Savani et al. 2017a,b). This averaging procedure must keep invariant the entropy production, a necessary condition listed already in the 1990s (Hassanizadeh and Gray 1990; Gray and Miller 2005).

\section{The Volumetric Flow Rate $Q$ is an Euler Homogeneous Function of Order One}

The volumetric flow rate $Q$ is a homogeneous function of order one of the areas $A_{w}$ and $A_{n}$ defined in Sect. 2. We now proceed to demonstrate this. Suppose we scale the three areas 


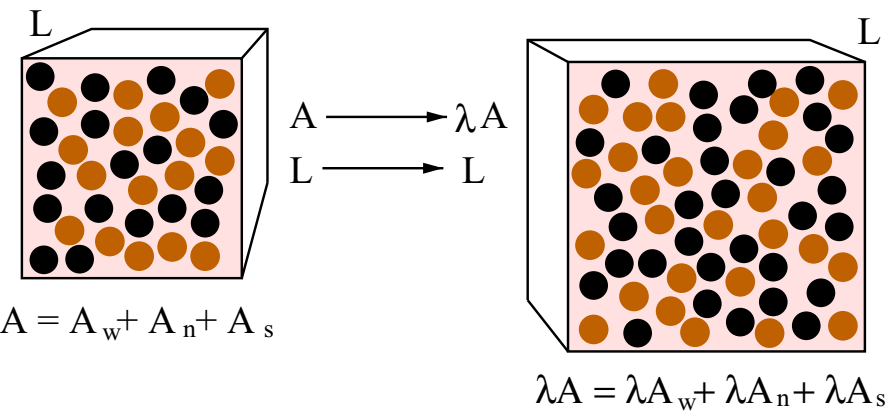

Fig. 2 A representative elementary volume (REV) is shown to the left. The flow direction is orthogonal to the figure plane. The REV has a length $L$ in the flow direction and an area $A$ orthogonal to the flow direction. That area may be split into three: $A_{S}$ which is the area of the solid matrix (colored pink), $A_{w}$ the area covered by pores filled with wetting fluid (colored brown) and $A_{n}$ the area covered by pores filled with non-wetting fluid (colored black). To the right, we show a different REV with an area $\lambda A$ orthogonal to the flow direction. The size of the pores in this REV is the same as in the one to the left as we assume them to be made of the same porous material. The solid matrix, wetting and non-wetting areas in this REV are then $\lambda A_{s}, \lambda A_{w}$ and $\lambda A_{n}$, respectively. The length of the REV is the same as the one to the left, $L$. Keeping the local (intensive) parameters of the flow fixed when rescaling the areas leads to a change in the volumetric flow rate from $Q$ (left) to $\lambda Q$ (right). This illustrates the contents of Eq. (15)

$A_{w} \rightarrow \lambda A_{w}, A_{n} \rightarrow \lambda A_{n}$ and $A_{s} \rightarrow \lambda A_{s}$, keeping in mind that

$$
A_{s}=\left(\frac{1}{\phi}-1\right) A_{p}=\left(\frac{1}{\phi}-1\right)\left(A_{w}+A_{n}\right),
$$

which we get from Eqs. (2) and (3). ${ }^{1}$ This corresponds to enlarging the area $A$ of the REV to $\lambda A$ as illustrated in Fig. 2. This transformation does not change the porosity $\phi$. Due to Eq. (14) with $\phi$ kept fixed, the only two independent variables in the problem are then $A_{w}$ and $A_{n}$, and as a consequence the volumetric flow rate depends on these two variables and not on $A_{s}, Q=Q\left(A_{w}, A_{n}\right)$. The scaling $A_{w} \rightarrow \lambda A_{w}$ and $A_{n} \rightarrow \lambda A_{n}$ affects the volumetric flow rate as follows:

$$
Q\left(\lambda A_{w}, \lambda A_{n}\right)=\lambda Q\left(A_{w}, A_{n}\right) .
$$

As long as the porous material does not have a fractal structure, this scaling property is essentially self-evident as explained in Fig. 2.

We take the derivative with respect to $\lambda$ on both sides of (15) and set $\lambda=1$. This gives ${ }^{2}$

$$
Q\left(A_{w}, A_{n}\right)=A_{w}\left(\frac{\partial Q}{\partial A_{w}}\right)_{A_{n}}+A_{n}\left(\frac{\partial Q}{\partial A_{n}}\right)_{A_{w}} .
$$

Equation (16) is essentially the Euler theorem for homogeneous functions of order one. By dividing this equation by $A_{p}$, we have

$$
v=S_{w}\left(\frac{\partial Q}{\partial A_{w}}\right)_{A_{n}}+S_{n}\left(\frac{\partial Q}{\partial A_{n}}\right)_{A_{w}},
$$

where we have used Eqs. (6) and (7).

\footnotetext{
${ }^{1}$ We do not scale the length of the REV $L$. By only scaling the area $A$ orthogonal to the flow direction and, hence, to the driving forces generating the flow, the relations between the driving forces and the flow are not affected. For this reason, the driving forces never enter the discussion explicitly.

${ }^{2}$ We clarify the physical meaning of these derivatives through a number of examples in Sect. 7.
} 
The two partial derivatives in Eq. (17) have the units of velocity. Hence, we define two thermodynamic velocities $\hat{v}_{w}$ and $\hat{v}_{n}$ as

$$
\hat{v}_{w}=\left(\frac{\partial Q}{\partial A_{w}}\right)_{A_{n}},
$$

and

$$
\hat{v}_{n}=\left(\frac{\partial Q}{\partial A_{n}}\right)_{A_{w}}
$$

Equation (17) then becomes

$$
v=S_{w} \hat{v}_{w}+S_{n} \hat{v}_{n} .
$$

The fact that the volumetric flow rate $Q$ is a homogeneous function of order one in the variables $A_{w}$ and $A_{n}$ implies that the thermodynamic velocities defined in Eqs. (18) and (19) must be homogeneous of the zeroth order so that we have

$$
\begin{aligned}
& \hat{v}_{w}\left(\lambda A_{w}, \lambda A_{n}\right)=\hat{v}_{w}\left(A_{w}, A_{n}\right), \\
& \hat{v}_{n}\left(\lambda A_{w}, \lambda A_{n}\right)=\hat{v}_{n}\left(A_{w}, A_{n}\right), \\
& v\left(\lambda A_{w}, \lambda A_{n}\right)=v\left(A_{w}, A_{n}\right) .
\end{aligned}
$$

This implies that they must depend on the areas $A_{w}$ and $A_{n}$ through their ratio $A_{w} / A_{n}=$ $S_{w} / S_{n}=S_{w} /\left(1-S_{w}\right)$, where we have used Eqs. (6)-(8) and we may write

$$
\begin{aligned}
& \hat{v}_{w}=\hat{v}_{w}\left(S_{w}\right), \\
& \hat{v}_{n}=\hat{v}_{n}\left(S_{w}\right), \\
& v=v\left(S_{w}\right) .
\end{aligned}
$$

\section{Consequences of the Euler Theorem: New Equations}

We now change the variables from $\left(A_{w}, A_{n}\right)$ to $\left(S_{w}, A_{p}\right)$ where $A_{w}=S_{w} A_{p}$ and $A_{n}=$ $\left(1-S_{w}\right) A_{p}$. We calculate

$$
\begin{aligned}
\left(\frac{\partial Q}{\partial S_{w}}\right)_{A_{p}} & =\left(\frac{\partial Q}{\partial A_{w}}\right)_{A_{n}}\left(\frac{\partial A_{w}}{\partial S_{w}}\right)_{A_{p}}+\left(\frac{\partial Q}{\partial A_{n}}\right)_{A_{w}}\left(\frac{\partial A_{n}}{\partial S_{w}}\right)_{A_{p}} \\
& =A_{p}\left[\hat{v}_{w}-\hat{v}_{n}\right] .
\end{aligned}
$$

We divide by the area $A_{p}$ and find

$$
\frac{\mathrm{d} v}{\mathrm{~d} S_{w}}=\hat{v}_{w}-\hat{v}_{n}
$$

replacing the partial derivative by a total derivative in accordance with Eq. (22).

We then proceed by taking the derivative of Eq. (20) with respect to $S_{w}$,

$$
\frac{\mathrm{d} v}{\mathrm{~d} S_{w}}=\frac{\mathrm{d}}{\mathrm{d} S_{w}}\left[S_{w} \hat{v}_{w}+\left(1-S_{w}\right) \hat{v}_{n}\right]=\hat{v}_{w}-\hat{v}_{n}+S_{w} \frac{\mathrm{d} \hat{v}_{w}}{\mathrm{~d} S_{w}}+\left(1-S_{w}\right) \frac{\mathrm{d} \hat{v}_{n}}{\mathrm{~d} S_{w}} .
$$

Combining this equation with Eq. (24), we find

$$
S_{w} \frac{\mathrm{d} \hat{v}_{w}}{\mathrm{~d} S_{w}}+\left(1-S_{w}\right) \frac{\mathrm{d} \hat{v}_{n}}{\mathrm{~d} S_{w}}=0 .
$$


This equation is a Gibbs-Duhem-like equation, here relating the thermodynamic velocities $\hat{v}_{w}$ and $\hat{v}_{n}$.

We note that Eqs. (20), (24) and (26) are interrelated in that any pair selected from the three equations will contain the third.

The thermodynamic velocities (18) and (19) may be expressed in terms of the variables $A_{p}$ and $S_{w}$. By using Eq. 12), i.e., $Q=A_{p} v$, and that $v$ is a function of $S_{w}$ only, see Eq. (22), we find

$$
\begin{aligned}
\hat{v}_{w} & =\left(\frac{\partial Q}{\partial A_{w}}\right)_{A_{n}}=\left(\frac{\partial A_{p} v}{\partial A_{p}}\right)_{S_{w}}\left(\frac{\partial A_{p}}{\partial A_{w}}\right)_{A_{n}}+\left(\frac{\partial A_{p} v}{\partial S_{w}}\right)_{A_{p}}\left(\frac{\partial S_{w}}{\partial A_{w}}\right)_{A_{n}} \\
& =v+S_{n} \frac{\mathrm{d} v}{\mathrm{~d} S_{w}}
\end{aligned}
$$

where we have used that

$$
\left(\frac{\partial S_{w}}{\partial A_{w}}\right)_{A_{n}}=\frac{1}{A_{p}}-\frac{A_{w}}{A_{p}^{2}}=\frac{S_{n}}{A_{p}} .
$$

Likewise, we find

$$
\hat{v}_{n}=\left(\frac{\partial Q}{\partial A_{n}}\right)_{A_{w}}=v-S_{w} \frac{\mathrm{d} v}{\mathrm{~d} S_{w}} .
$$

By taking the derivative with respect to $S_{w}$ of Eqs. (27) and (29), we find

$$
\frac{\mathrm{d} \hat{v}_{w}}{\mathrm{~d} S_{w}}=S_{n} \frac{\mathrm{d}^{2} v}{\mathrm{~d} S_{w}^{2}},
$$

and

$$
\frac{\mathrm{d} \hat{v}_{n}}{\mathrm{~d} S_{w}}=-S_{w} \frac{\mathrm{d}^{2} v}{\mathrm{~d} S_{w}^{2}} .
$$

\subsection{New Equations in Terms of the Seepage Velocities}

Equation (20) has the same form as Eq. (13),

$$
v=S_{w} v_{w}+S_{n} v_{n}=S_{w} \hat{v}_{w}+S_{n} \hat{v}_{n} .
$$

This does not imply that $\hat{v}_{w}=v_{w}$ and $\hat{v}_{n}=v_{n}$. The most general relations we can write down between $\hat{v}_{w}$ and $v_{w}$, and $\hat{v}_{n}$ and $v_{n}$ and still fulfill (32) are

$$
\hat{v}_{w}=v_{w}+S_{n} v_{m}\left(S_{w}\right)
$$

and

$$
\hat{v}_{n}=v_{n}-S_{w} v_{m}\left(S_{w}\right),
$$

where $v_{m}$ is a function of $S_{w}$ with the units of velocity. We name this function the co-moving velocity. We may interpret the physical contents of the second equality in (32) in the following way: The thermodynamic velocities $\hat{v}_{w}$ and $\hat{v}_{n}$ differ from the $v_{w}$ and $v_{n}$ as each fluid carries along some of the other fluid-hence, "co-moving." To preserve the volume, these co-moving contributions are related in such a way that $A_{n} S_{w} v_{m}$ is the co-moving volume flow of the non-wetting fluid with the wetting fluid and $A_{w} S_{n} v_{m}$ is the co-moving volume flow of the wetting fluid with the non-wetting fluid. 
By using Eqs. (33) and (34), we find that the seepage velocities (9) and (10) also are homogeneous of the zeroth order so that

$$
\begin{aligned}
& v_{w}=v_{w}\left(S_{w}\right), \\
& v_{n}=v_{n}\left(S_{w}\right) .
\end{aligned}
$$

Equations (20), (24) and (26) are in terms of the thermodynamic velocities defined by the area derivatives of the volumetric flow, (18) and (19). We now express them in terms of the seepage velocities $v_{w}$ and $v_{n}$ defined in Eqs. (9) and (10). We do this by invoking transformations (33) and (34). Equation (24) then becomes

$$
\frac{\mathrm{d} v}{\mathrm{~d} S_{w}}=v_{w}-v_{n}+v_{m},
$$

where we have used Eqs. (33) and (34) and $v_{m}$ is the co-moving velocity.

Expressing (26) in terms of the seepage velocities (9) and (10), we find

$$
S_{w} \frac{\mathrm{d} v_{w}}{\mathrm{~d} S_{w}}+\left(1-S_{w}\right) \frac{\mathrm{d} v_{n}}{\mathrm{~d} S_{w}}=v_{m} .
$$

The three Eqs. (13), (36) and (37) are dependent: Combine any two of them and the third will follow.

By a derivation similar to that which led to Eqs. (30) and (31), we find

$$
\frac{\mathrm{d} v_{w}}{\mathrm{~d} S_{w}}=v_{m}+S_{n}\left[\frac{\mathrm{d}^{2} v}{\mathrm{~d} S_{w}^{2}}-\frac{\mathrm{d} v_{m}}{\mathrm{~d} S_{w}}\right],
$$

and

$$
\frac{\mathrm{d} v_{n}}{\mathrm{~d} S_{w}}=v_{m}-S_{w}\left[\frac{\mathrm{d}^{2} v}{\mathrm{~d} S_{w}^{2}}-\frac{\mathrm{d} v_{m}}{\mathrm{~d} S_{w}}\right]
$$

\section{Cross Points}

We may define three wetting saturations of special interest, $S_{w}^{A}, S_{w}^{B}$ and $S_{w}^{C}$, that have the following properties:

- $A$ When $S_{w}=S_{w}^{A}$ we have $v_{w}=\hat{v}_{w}$ and $v_{n}=\hat{v}_{n}$. By Eqs. (33) and (34), we have that $v_{m}=0$ for this wetting saturation. We furthermore have that $v_{m} \leq 0$ for $S_{w} \leq S_{w}^{A}$ and $v_{m} \geq 0$ for $S_{w} \geq S_{w}^{A}$.

- $B$ When $S_{w}=S_{w}^{B}$, we have that $\hat{v}_{w}=\hat{v}_{n}$. From Eq. (32), we find $\hat{v}_{w}=\hat{v}_{n}=v$. Equations (27) and (29) make $\mathrm{d} v / \mathrm{d} S_{w}=0$ equivalent to the equality between the three velocities at this wetting saturation. Furthermore, at $\mathrm{d} v / \mathrm{d} S_{w}=0, v$ has its minimum value. We have that $\mathrm{d} v / \mathrm{d} S_{w} \leq 0$ for $S_{w} \leq S_{w}^{B}$ and $\mathrm{d} v / \mathrm{d} S_{w} \geq 0$ for $S_{w} \geq S_{w}^{B}$.

- $C$ When $S_{w}=S_{w}^{C}$, we have that $v_{w}=v_{n}$. This implies that $v_{w}=v_{n}=v$ from Eq. (13) for this wetting saturation. By Eqs. (42) and (43), we have that this is equivalent to $v_{m}=\mathrm{d} v / \mathrm{d} S_{w}$.

By considering the relations between the velocity variables derived so far, we may show that we either have

$$
S_{w}^{A} \leq S_{w}^{B} \leq S_{w}^{C},
$$

or

$$
S_{w}^{C} \leq S_{w}^{B} \leq S_{w}^{A} .
$$


It is straightforward to demonstrate that if either two of the three wetting saturations $S_{w}^{A}$, $S_{w}^{B}$ or $S_{w}^{C}$ coincide, then the third must also have the same value. If this is the case, we have $v_{w}=v_{n}$ and $\mathrm{d} v / \mathrm{d} S_{w}=0$, so that the two immiscible fluids behave as if they were miscible (Sinha et al. 2018). We will use our theory to identify the wetting saturation at which this coincidence occurs for the network model we study in Sect. 8 and then verify numerically that this is indeed so.

\section{A Closed Set of Equations}

We now consider the porous medium on scales larger than the REV scale. The properties of the porous medium may at these scales vary in space. We consider here only flow in the $x$ direction, deferring the generalization to higher dimensions to later. Let $x$ be a point somewhere in this porous medium. Hence, all the variables in the following will be functions of $x$.

Equations (13) and (37),

$$
\begin{aligned}
v & =S_{w} v_{w}+S_{n} v_{n}, \\
v_{m} & =S_{w} v_{w}^{\prime}+S_{n} v_{n}^{\prime},
\end{aligned}
$$

where $v_{w}^{\prime}=\mathrm{d} v_{w} / \mathrm{d} S_{w}$ and $v_{n}^{\prime}=\mathrm{d} v_{n} / \mathrm{d} S_{w}$, can be seen as a transformation from the velocity pair $\left(v_{w}, v_{n}\right)$ to the pair $\left(v, v_{m}\right)$. We invert the velocity transformation, $\left(v, v_{m}\right) \rightarrow\left(v_{w}, v_{n}\right)$, to find

$$
v_{w}=v+S_{n}\left(v^{\prime}-v_{m}\right)
$$

and

$$
v_{n}=v-S_{w}\left(v^{\prime}-v_{m}\right)
$$

where $v^{\prime}=\mathrm{d} v / \mathrm{d} S_{w}$.

We now turn to the equations controlling the time evolution of the flow. The transport equation for $S_{w}$ is

$$
\frac{\partial S_{w}}{\partial t}+\frac{\partial S_{w} v_{w}}{\partial x}=0
$$

Likewise, we have for $S_{n}$

$$
\frac{\partial S_{n}}{\partial t}+\frac{\partial S_{n} v_{n}}{\partial x}=0 .
$$

Summing these two equations and using Eqs. (5) and (13), we find the incompressibility condition

$$
\frac{\partial v}{\partial x}=0 .
$$

Equations (13), (37), (44) and (46) constitute four equations. There are five variables: $v$, $v_{m}, v_{w}, v_{n}$ and $S_{w}$. Seemingly, we are one equation short of a closed set.

In order to close the equation set, we need an equation for the co-moving velocity $v_{m}$. This is a constitutive equation that characterizes how the immiscible fluids affect each other due to the constraints imposed by the porous matrix,

$$
v_{m}=v_{m}\left(S_{w}, v, v^{\prime}\right) .
$$

Specifying $v_{m}$ will specify the form of the differential equation (37). We will work out $v_{m}$ analytically for four systems in Sect. 7 and map it for a range of parameters in a network 
model in Sect. 8. This numerical mapping is based on the Gibbs-Duhem equation, (37). This would also be the way $v_{m}$ is measured in the laboratory.

Note that it is not enough to specify $S_{w}$ and $v$ to determine $v_{m}$. It is also a function of $\mathrm{d} v / \mathrm{d} S_{w}$, since $\mathrm{d} v / \mathrm{d} S_{w}$ depends on how the external parameters are controlled as $S_{w}$ is changed. For example, if the total volumetric flow rate $Q=A_{p} v$ is held constant when $S_{w}$ is changed, then $\mathrm{d} v / \mathrm{d} S_{w}=0$ for all values of $S_{w}$, and the system traces the curve $\left(S_{w}, v, v^{\prime}\right)$ space so that $v^{\prime}=0$. If, on the other hand, the driving forces are held constant when $S_{w}$ is changed, then $v$ and $\mathrm{d} v / \mathrm{d} S_{w}$ will follow some non-trivial curve in $\left(S_{w}, v, v^{\prime}\right)$ space; see Sect. 8. A third example is to control the wetting and the non-wetting volumetric flow rates $Q_{w}$ and $Q_{n}$, making $S_{w}$ and $v$ and $\mathrm{d} v / \mathrm{d} S_{w}$ dependent variables, following curves in $\left(S_{w}, v, v^{\prime}\right)$ space depending on how $Q_{w}$ and $Q_{n}$ are changed.

The driving forces enter the discussion through the constitutive equation

$$
v=v\left(S_{w}, f\right)
$$

where $f$ represents the driving forces, for example, $f=-\partial P / \partial x$, i.e., minus the pressure gradient.

The equation set (13), (37), (44) and (46) together with the constitutive equation for $v_{m}$ is independent of the driving forces. They are valid for any constitutive Eq. (48) that may be proposed. In particular, nonlinear constitutive equations such as those recently proposed which suggest that two immiscible fluids behave as if they were a single Bingham plastic (Tallakstad et al. 2009a, b; Rassi et al. 2011; Sinha and Hansen 2012; Sinha et al. 2017) fit well into this framework.

This is in stark contrast to, for example, the relative permeability framework that assumes a particular constitutive equation for $v_{w}$ and $v_{n}$ containing the two relative permeabilities $k_{r, w}\left(S_{w}\right)$ and $k_{r, n}\left(S_{w}\right)$ in addition to the capillary pressure function $P_{c}\left(S_{w}\right)$. The equation set we present here is thus much more general than that of the relative permeability framework.

\section{Analytically Tractable Models}

We will in this section analyze four variants of the capillary tube model (Scheidegger 1953, 1974). In each case, we calculate the co-moving velocity $v_{m}$ and then use it to demonstrate the consistency of our theory. We also use this section to clarify the physical meaning of the area derivatives introduced in Sect. 3.

\subsection{Parallel Capillaries Filled with Either Fluid}

In this simplest case, we envision a bundle of $N$ parallel capillaries, all equal. Each tube has a cross-sectional inner (i.e., pore) area $a_{p} . N_{w}$ of these capillaries are filled with the wetting fluid, and $N_{n}$ are filled with the non-wetting fluid, so that $N_{w}+N_{n}=N$. Let us assume that the seepage velocity in the capillaries filled with wetting fluid is $v_{w}$ and the seepage velocity in the capillaries filled with non-wetting fluid is $v_{n}$. The total volumetric flow rate is then

$$
Q=N_{w} a_{p} v_{w}+N_{n} a_{p} v_{n} .
$$

The wetting saturation is given by

$$
S_{w}=\frac{A_{w}}{A_{p}}=\frac{N_{w}}{N} .
$$


We calculate the derivative of $Q$ with respect to $A_{w}$ to determine the thermodynamic velocity $\hat{v}_{w}$ defined in Eq. (18) together with Eq. (49),

$$
\hat{v}_{w}=\left(\frac{\partial Q}{\partial A_{w}}\right)_{A_{n}}=\frac{1}{a_{p} \delta N_{w}}\left[Q\left(N_{w}+\delta N_{w}, N_{n}\right)-Q\left(N_{w}, N_{n}\right)\right]=v_{w} .
$$

The derivative is thus performed changing the number of capillaries from $N$ to $N+\delta N$ and letting all the added capillaries contain the wetting fluid so that $N_{w} \rightarrow N_{w}+\delta N_{w}=N_{w}+\delta N$. Likewise, we find using Eq. (19)

$$
\hat{v}_{n}=\left(\frac{\partial Q}{\partial A_{n}}\right)_{A_{w}}=\frac{1}{a_{p} \delta N_{n}}\left[Q\left(N_{w}, N_{n}\right)-Q\left(N_{w}, N_{n}+\delta N_{n}\right)\right]=v_{n} .
$$

From Eqs. (33) and (34), we find that the co-moving velocity is zero,

$$
v_{m}=0
$$

for this system. Hence, we have that the seepage velocities $v_{w}$ and $v_{n}$ are equal to the thermodynamic velocities $\hat{v}_{w}$ and $\hat{v}_{n}$.

\subsection{Parallel Capillaries with Bubbles}

Suppose that we have $N$ parallel capillaries as shown in Fig. 3. Each tube has a length $L$ and an average inner area $a_{p}$. The diameter of each capillary varies along the long axis. Each capillary is filled with a bubble train of wetting or non-wetting fluid. The capillary forces due to the interfaces vary as the bubble train moves due to the varying diameter. We furthermore assume that the wetting fluid, or more precisely the more wetting fluid (in contrast to the non-wetting fluid which is the less wetting fluid) does not form films along the pore walls so that the fluids do not pass each other; see Sinha et al. (2013) for details. The volume of the wetting fluid in each tube is $L_{w} a_{p}$, and the volume of the non-wetting fluid is $L_{n} a_{p}$. Hence, the saturations are $S_{w}=L_{w} / L$ and $S_{n}=L_{n} / L$ for each tube.

Suppose now that the seepage velocity in each tube is $v$ when averaged over time. Both the wetting and non-wetting seepage velocities must be equal to the average seepage velocity since the bubbles do not pass each other:

$$
v_{w}=v_{n}=v .
$$

We now make an imaginary cut through the capillaries orthogonal to the flow direction as shown in Fig. 3. There will be a number $N_{w}$ capillaries where the cut passes through the wetting fluid and a number $N_{n}$ capillaries where the cut passes through the non-wetting fluid. Averaging over time, we must have

$$
\frac{\left\langle N_{w}\right\rangle}{N}=\frac{L_{w}}{L}=S_{w}
$$

and

$$
\frac{\left\langle N_{n}\right\rangle}{N}=\frac{L_{n}}{L}=S_{n} .
$$

Hence, the wetting and non-wetting areas defined in Sect. 2 are

$$
A_{w}=\left\langle N_{w}\right\rangle a_{p}
$$

and

$$
A_{n}=\left\langle N_{n}\right\rangle a_{p} ; .
$$




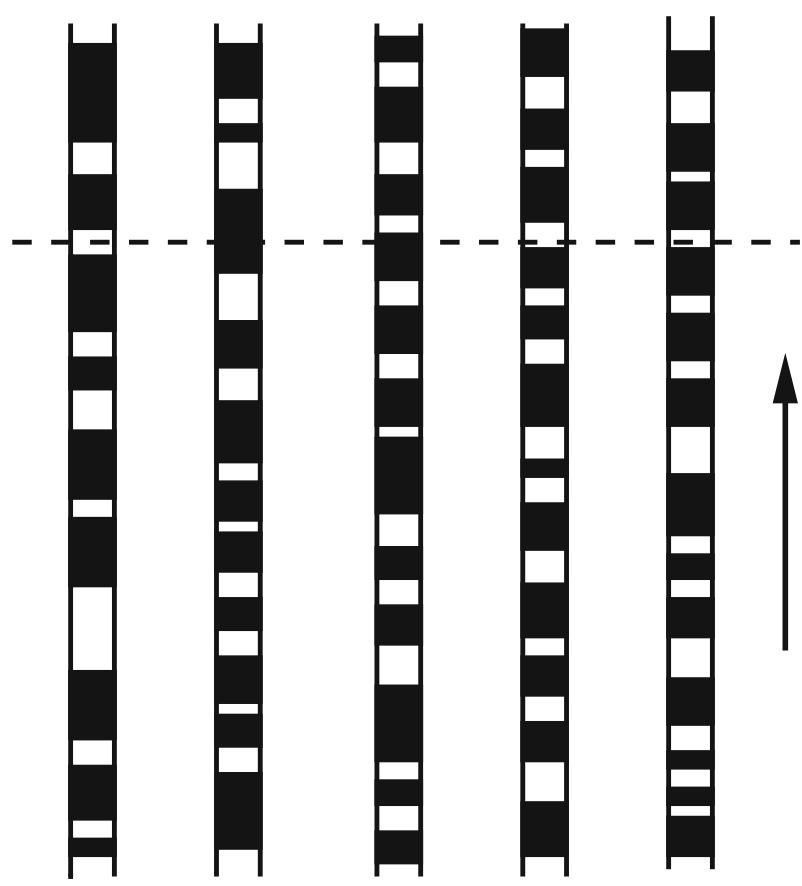

Fig. 3 Parallel tube model analyzed in Sect. 7.2. There are $N=5$ capillaries, each filled with a bubble train of wetting (white) and non-wetting (black) fluid moving in the direction of the arrow. The diameter along each tube varies so that the capillary force from each interface varies with its position. The varying diameters are not illustrated in the figure; only the average diameter is shown. This is the same for all of the capillaries. Each capillary has a length $L$ and an inner area $a_{p}$. The wetting fluid in each tube occupies a volume $L_{w} a_{p}$, whereas the non-wetting fluid occupies a volume $L_{n} a_{p}$. The broken line illustrates the imaginary cut through the capillary tube bundle

We also have that

$$
A_{p}=N a_{p} .
$$

We will now calculate derivative (18) defining the thermodynamic velocity $\hat{v}_{w}$. We do this by changing the pore area $A_{p} \rightarrow A_{p}+\delta A_{p}=N a_{p}+a_{p} \delta N$. We wish to change $A_{w}$ while keeping $A_{n}$ fixed. This can only be done by adjusting $S_{w}$ while changing $\delta N$. This leads to the two equations

$$
\delta\left\langle N_{w}\right\rangle=\delta\left[N S_{w}\right]=N \delta S_{w}+S_{w} \delta N=\delta N
$$

and

$$
\delta\left\langle N_{n}\right\rangle=\delta\left[N\left(1-S_{w}\right)\right]=-N \delta S_{w}+\left(1-S_{w}\right) \delta N=0 .
$$

We solve either (60) or (61) for $\delta S_{w}$ (they contain the same information), finding

$$
\delta S_{w}=\frac{\left(1-S_{w}\right)}{N} \delta N
$$

Hence, we have

$$
\hat{v}_{w}=\left(\frac{\partial Q}{\partial A_{w}}\right)_{A_{n}}=\frac{1}{\delta A_{p}}\left[\left(A_{p}+\delta A_{p}\right) v\left(S_{w}+\delta S_{w}\right)-A_{p} v\left(S_{w}\right)\right]
$$




$$
\begin{aligned}
& =\frac{1}{\delta N}\left[(N+\delta N) v\left(S_{w}+\delta S_{w}\right)-N v\left(S_{w}\right)\right] \\
& =\frac{1}{\delta N}\left[(N+\delta N)\left[v\left(S_{w}\right)+\left(\mathrm{d} v / \mathrm{d} S_{w}\right) \delta S_{w}\right]-N v\left(S_{w}\right)\right] \\
& =\frac{1}{\delta N}\left[(N+\delta N)\left[v\left(S_{w}\right)+\left(1-S_{w}\right)\left(\mathrm{d} v / \mathrm{d} S_{w}\right)(\delta N / N)\right]-N v\left(S_{w}\right)\right] \\
& =v\left(S_{w}\right)+S_{n} \frac{\mathrm{d} v\left(S_{w}\right)}{\mathrm{d} S_{w}} .
\end{aligned}
$$

We recognize that this equation is Eq. (27). Hence, we could have taken this equation as the starting point of discussing this model. However, doing the derivative explicitly demonstrates its operational meaning.

Rather than performing a similar calculation for $\hat{v}_{n}$ as in (63) for $\hat{v}_{w}$, we use Eq. (29) and have

$$
\hat{v}_{n}=\left(\frac{\partial Q}{\partial A_{n}}\right)_{A_{w}}=v\left(S_{w}\right)-S_{w} \frac{\mathrm{d} v\left(S_{w}\right)}{\mathrm{d} S_{w}} .
$$

We now combine Eqs. (54), (63) and (64) with (33) and (34) and read off

$$
v_{m}=\frac{\mathrm{d} v}{\mathrm{~d} S_{w}} .
$$

We see that the expression for $v_{m}$ is independent of the constitutive equation that relates flow rate to the driving forces. It expresses that both fluid species are forced to move with the same seepage velocity, whereas the thermodynamic velocities remain different for the two fluids.

Combining Eq. (65) for $v_{m}$ with Eqs. (33) and (34) gives Eq. (54) as it must.

This result gives us an opportunity to clarify the physical meaning of the co-moving velocity $v_{m}$. We have assumed that the bubbles cannot pass each other as shown in Fig. 3. Equation (65) gives upon substitution in Eqs. (33) and (34),

$$
v_{w}=\hat{v}_{w}-S_{n} \frac{\mathrm{d} v}{\mathrm{~d} S_{w}},
$$

and

$$
v_{n}=\hat{v}_{n}+S_{w} \frac{\mathrm{d} v}{\mathrm{~d} S_{w}} .
$$

We see that these two equations compensate exactly for the two Eqs. (27) and (29),

$$
\begin{aligned}
& \hat{v}_{w}=v+S_{n} \frac{\mathrm{d} v}{\mathrm{~d} S_{w}}, \\
& \hat{v}_{n}=v-S_{w} \frac{\mathrm{d} v}{\mathrm{~d} S_{w}},
\end{aligned}
$$

that give the thermodynamic velocities resulting in $v_{w}=v_{n}=v$.

\subsection{Parallel Capillaries with a Subset of Smaller Ones}

We now turn to the third example. There are $N$ parallel capillaries of length $L$. A fraction of these capillaries has an inner area $a_{s}$, whereas the rest has an inner area $a_{l}$. The total pore area carried by the small capillaries is $A_{s}$ and the total pore area carried by the larger capillaries 
is $A_{l}$ so that

$$
A_{p}=A_{s}+A_{l},
$$

We define the fraction of small capillaries to be $S_{w, i}$, so that

$$
A_{s}=S_{w, i} A_{p} .
$$

We now assume that the small capillaries are so narrow that only the wetting fluid enters them. These pores constitute the irreducible wetting fluid contents of the model in that we cannot go below this saturation. However, they still contribute to the flow. Hence, at a saturation $S_{w}$ which we assume to be larger than or equal to $S_{w, i}$ which is then the irreducible wetting fluid saturation, we have that the wetting pore area is

$$
A_{w}=S_{w, i} A_{p}+\left(S_{w}-S_{w, i}\right) A_{p}=A_{s}+A_{l w},
$$

where we have defined $A_{l w}=\left(S_{w}-S_{w, i}\right) A_{p}$.

We assume there is a seepage velocity $v_{s w}$ in the small capillaries, a seepage velocity $v_{l w}$ in the larger capillaries filled with wetting fluid and a seepage velocity $v_{n}$ in the larger filled with non-wetting fluid. The velocities $v_{s w}, v_{l w}$ and $v_{n}$ are independent of each other. The total volumetric flow rate is then given by

$$
Q=A_{s} v_{s w}+A_{l w} v_{l w}+A_{n} v_{n},
$$

or in terms of average seepage velocity

$$
v=S_{w, i} v_{s w}+\left(S_{w}-S_{w, i}\right) v_{l w}+S_{n} v_{n} .
$$

We will now calculate derivative (18) defining the thermodynamic velocity $\hat{v}_{w}$. We could have done this using Eq. (27). However, it is instructive to perform the derivative yet again through area differentials so that their meaning becomes clear.

In order to calculate derivative (19), we change the pore area $A_{p} \rightarrow A_{p}+\delta A_{p}$ so that $\delta A_{w}=\delta A_{p}$ and $\delta A_{n}=0$. In addition, we have that $\delta A_{s}=S_{w, i} \delta A_{p}$, so that

$$
\delta A_{w}=\delta A_{s}+\delta A_{l w}=S_{w, i} \delta A_{p}+\delta A_{l w}=\delta A_{p} .
$$

Hence, we have

$$
\delta A_{l w}=\left(1-S_{w, i}\right) \delta A_{p} .
$$

We now combine this expression with Eq. (71) for the total volumetric flow $Q$ to find

$$
\begin{aligned}
\hat{v}_{w} & =\left(\frac{\partial Q}{\partial A_{w}}\right)_{A_{n}}=\frac{1}{\delta A_{p}}\left[\delta A_{s} v_{s w}+\delta A_{l w} v_{l w}\right] \\
& =v_{l w}+S_{w, i}\left(v_{s w}-v_{l w}\right) .
\end{aligned}
$$

We could have found $\hat{v}_{n}$ in the same way. However, using (29) combined with (72) gives

$$
\hat{v}_{n}=v_{n}+S_{w, i}\left(v_{s w}-v_{l w}\right) .
$$

We now use Eq. (34) to find

$$
\hat{v}_{n}-v_{n}=-v_{m} S_{w}=S_{w, i}\left(v_{s w}-v_{l w}\right)=-\left[\frac{S_{w, i}}{S_{w}}\left(v_{l w}-v_{s w}\right)\right] S_{w},
$$

so that

$$
v_{m}=\frac{S_{w, i}}{S_{w}}\left(v_{l w}-v_{s w}\right) .
$$


As a check, we now use the co-moving velocity found in (78) together with Eqs. (75) and (33) to calculate $v_{w}$. We find

$$
v_{w}=\hat{v}_{w}-v_{m} S_{n}=\frac{1}{S_{w}}\left[S_{w, i} v_{s w}+\left(S_{w}-S_{w, i}\right) v_{l w}\right],
$$

which is the expected result; see Eq. (72).

\subsection{Large Capillaries with Bubbles and Small Capillaries with Wetting Fluid only}

The fourth example that we consider is a combination of the two previous models discussed in Sects. 7.2 and 7.3, namely a set of $N$ capillary tubes. A fraction $S_{w, i}$ of these capillaries has an inner area $a_{s}$, whereas the rest has an inner area $a_{l}$. The capillaries with the larger area contain bubbles as in Sect. 7.2. The smaller capillaries contain only wetting liquid as in Sect. 7.3. The fluid contents in the small capillaries are irreducible. The seepage velocity in the smaller capillaries is $v_{s w}$. The wetting and non-wetting seepage velocities in the larger capillaries are the same,

$$
v_{n}=v_{l w}=v_{l} .
$$

Hence, the seepage velocity is then

$$
v=S_{w, i} v_{s w}+\left(1-S_{w, i}\right) v_{l} .
$$

We have that

$$
\frac{\mathrm{d} v}{\mathrm{~d} S_{w}}=\left(1-S_{w, i}\right) \frac{\mathrm{d} v_{l}}{\mathrm{~d} S_{w}},
$$

since $v_{s w}$ is independent of $S_{w}$. Equation (29) gives

$$
\hat{v}_{n}=S_{w, i} v_{s w}+\left(1-S_{w, i}\right)\left(v_{l}-S_{w} \frac{\mathrm{d} v_{l}}{\mathrm{~d} S_{w}}\right),
$$

where we have used (81). We now use Eqs. (34) and (80) to find

$$
\hat{v}_{n}-v_{n}=\hat{v}_{n}-v_{l}=-S_{w} v_{m} .
$$

Combining Eqs. (82), (83) and (84) gives

$$
v_{m}=\frac{S_{w, i}}{S_{w}}\left(v_{l}-v_{s w}\right)+\frac{\mathrm{d} v}{\mathrm{~d} S_{w}} .
$$

Hence, the co-moving velocity is a sum of the co-moving velocities of the two previous sections; see (65) and (78).

We check the consistency of this calculation by using the co-moving velocity found in (85) together with Eq. (42) to calculate $v_{w}$. We find as expected

$$
v_{w}=\frac{1}{S_{w}}\left[S_{w, i} v_{s w}+\left(S_{w}-S_{w, i}\right) v_{l}\right] .
$$

These four analytically tractable examples have allowed us to demonstrate in detail how the thermodynamic formalism that we have introduced in this paper works. We have in particular calculated the co-moving velocity $v_{m}$ in all four cases. As is clear from these examples, it does not depend on the constitutive equation or any other equation except through $v$ and $\mathrm{d} v / \mathrm{d} S_{w}$.

Hence, the discussion of these four models has not included the constitutive Eq. (48). In order to have the seepage velocities $v_{w}$ and $v_{n}$ as functions of the driving forces, we 
would need to include the constitutive equation and the incompressibility condition (46) in the analysis.

\section{Network Model Studies}

Our aim in this section is to map the co-moving velocity $v_{m}$ for a network model which we describe in the following. We compare the measured seepage velocities with those calculated using the formulas derived earlier. We use the theory to predict where the three cross points defined and discussed in Sect. 5 coincide and verify this prediction through direct numerical calculation.

The network model first proposed by Aker et al. has been refined over the years and is today a versatile model for immiscible two-phase flow under steady-state conditions due to the implementation of bi-periodic boundary conditions. The model tracks the interfaces between the immiscible fluids by solving the Kirchhoff equations with a capillary pressure in links created by the interfaces they contain due to a surface tension $\sigma$. The links are hour glass shaped with average radii $r$ drawn from a probability distribution. The minimum size of the bubbles of wetting or non-wetting fluid in a given link has a minimum length of $r$, the average radius of that link.

Our network and flow parameters were chosen as follows: We used hexagonal (honeycomb) lattices of size $60 \times 40$ and square lattices of size $80 \times 30$, both with all links having the same length $l=1 \mathrm{~mm}$. The radii $r$ of the links were assigned from a flat distribution in the interval $0.1 l<r<0.4 l$. The surface tension $\sigma$ was either $0.02,0.03$ or $0.04 \mathrm{~N} / \mathrm{m}$. The viscosities of the fluids $\eta_{w}$ and $\eta_{n}$ were set to either $10^{-3}, 2 \times 10^{-3}, 5 \times 10^{-3}$ or $10^{-2} \mathrm{~Pa}$ s. The pressure drop over the width of the network $|\Delta P / L|$ was held constant during each run and set to either $0.1,0.15,0.20 .3,0.5$ or $1 \mathrm{MPa} / \mathrm{m}$. The capillary number Ca ranged from around $10^{-3}$ to $10^{-1}$; see Fig. 4 . We define the capillary number as

$$
\mathrm{Ca}=\frac{\mu_{n} Q}{\sigma A_{p}},
$$

where $\mu_{n}$ is viscosity of the non-wetting fluid. It was calculated in the network model at each time step as

$$
\mathrm{Ca}=\frac{\mu_{n} \sum_{j} \sum_{i} q_{i j}}{\sigma \sum_{j^{\prime}} \sum_{i^{\prime}} a_{i^{\prime} j^{\prime}}} .
$$

The sums are taken over all links $i$ in each layer $j$. The time-averaged value of $\mathrm{Ca}$ is obtained by averaging over all time steps, weighted by the time step length.

We measure the seepage velocities $v_{w}$ as follows. In both networks, we define layers normal to the flow direction. Each layer has an index $j$ and is the set of links intersected by a plane normal to the flow direction. In layer $j$, each link has an index $i$. For every time step, we calculate

$$
v_{w}=\frac{\sum_{j} \sum_{i} q_{w, i j}}{\sum_{j^{\prime}} \sum_{i^{\prime}} S_{w, i^{\prime} j^{\prime}} a_{i^{\prime} j^{\prime}}},
$$

where $S_{w, i j}$ is the saturation of link $i$ in layer $j$ and $a_{i j}$ is the cross-sectional area of the link, projected into the plane normal to the flow direction. The flow rate $q_{w, i j}$ is measured as the volume of wetting fluid that has flowed past the middle of the link during the time step, divided by the length of the time step. The time-averaged value of $v_{w}$ is then calculated by averaging over all time steps, weighted by the time step length. The non-wetting fluid velocities $v_{n}$ are measured in the same way. 


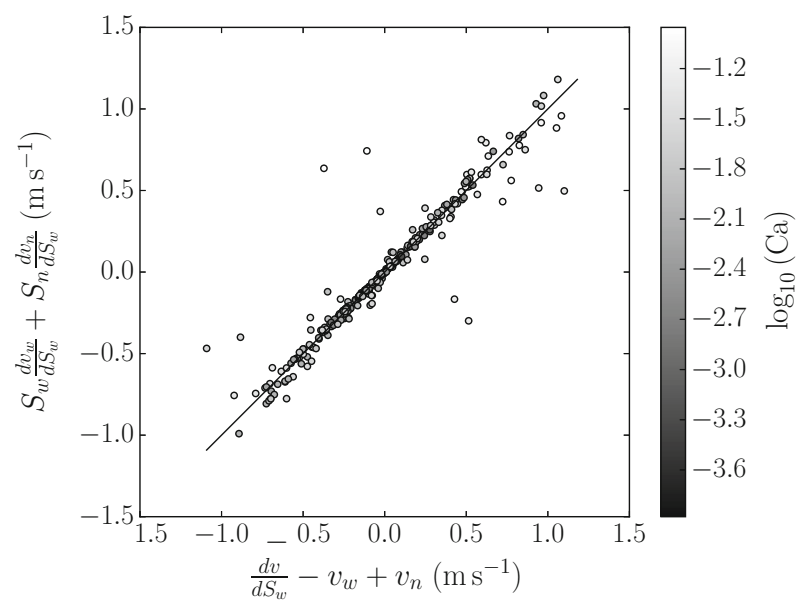

Fig. 4 Co-moving velocity $v_{m}$ may be expressed as in Eq. (36): $v_{m}=v^{\prime}-v_{w}+v_{n}$, or as in Eq. (37): $v_{m}=S_{w} v_{w}^{\prime}+S_{n} v_{n}^{\prime}$. We plot here the two different expressions for $v_{m}$ against each other. The data points are shaded according to the capillary number $\mathrm{Ca}$ of the flow. The data are based both on the hexagonal and the square lattice

We show in Fig. $4 v_{m}=v^{\prime}-v_{w}+v_{n}$ (Eq. (36) versus $v_{m}=S_{w} v_{w}^{\prime}+S_{n} v_{n}^{\prime}$ (Eq. 37). This verifies the main results of Sect. 4.1, besides being a measure of the quality of the numerical derivatives based on central difference for the internal points. Forward/backward difference was used at the endpoints of the curves where $S_{w}=0$ or $S_{w}=1$.

Figure 5 shows the co-moving velocity $v_{m}$ calculated from Eq. (37) as a function of $S_{w}$ and $v^{\prime}$ for the flow parameters given above. The data are roughly consistent with a linear form

$$
v_{m}=a S_{w}+b \frac{\mathrm{d} v}{\mathrm{~d} S_{w}}-c,
$$

where $a \approx-0.15, b \approx 0.79$ and $c \approx-0.095$. We note that we show both the data from the hexagonal and the square lattices in Fig. 5. The co-moving velocity $v_{m}$ appears not to be sensitive to the lattice topology.

We compare in Fig. 6 the measured seepage velocities $v_{w}$ and $v_{n}$ to the seepage velocities calculated from Eqs. (42) and (43) where we have used the co-moving velocity shown in Fig. 5. The spikes at low $v_{w}$ and $v_{n}$ are due to the transition from both fluids moving to only one fluid moving creating a jump in the derivative.

We note that when the wetting saturation $S_{w}$ is equal to $S_{w, 0}=c / a$ in Eq. (90), the comoving velocity $v_{m}$ takes the form $v_{m}=b v^{\prime}$. For the flows we study here, we find $S_{w, 0} \approx 0.6$. With reference to the discussion of the cross points in Sect. 5, we then have $v_{m}=0$ and $v^{\prime}=0$ occurring for the same wetting saturation. Hence, we have $S_{w}^{A}=S_{w}^{B}=S_{w}^{C}$ at this point.

Figure 5 shows two closely spaced curves, one dotted and one dashed. The dashed curve passes through the point $\left(v_{m}, v^{\prime}\right)=(0,0)$ (marked by a non-filled circle). We show in Fig. 7 $v, v_{w}, v_{n}, \hat{v}_{w}$ and $\hat{v}_{n}$ corresponding to these two curves. The thermodynamic velocities have been calculated using Eqs. (27) and (29). In Fig. 7a, the flow parameters have been chosen so that $v^{\prime}=0$ and $v_{m}=0$ do not coincide as we see in Fig. 5. The ordering of the cross points then follows inequality (41): $S_{w}^{C} \leq S_{w}^{B} \leq S_{w}^{A}$. In Fig. 7b, we have chosen the parameters so that $v^{\prime}=v_{m}=0$ for a given saturation, $S_{w}=S_{w, 0} \approx 0.6$; see Fig. 5 . The theory then 


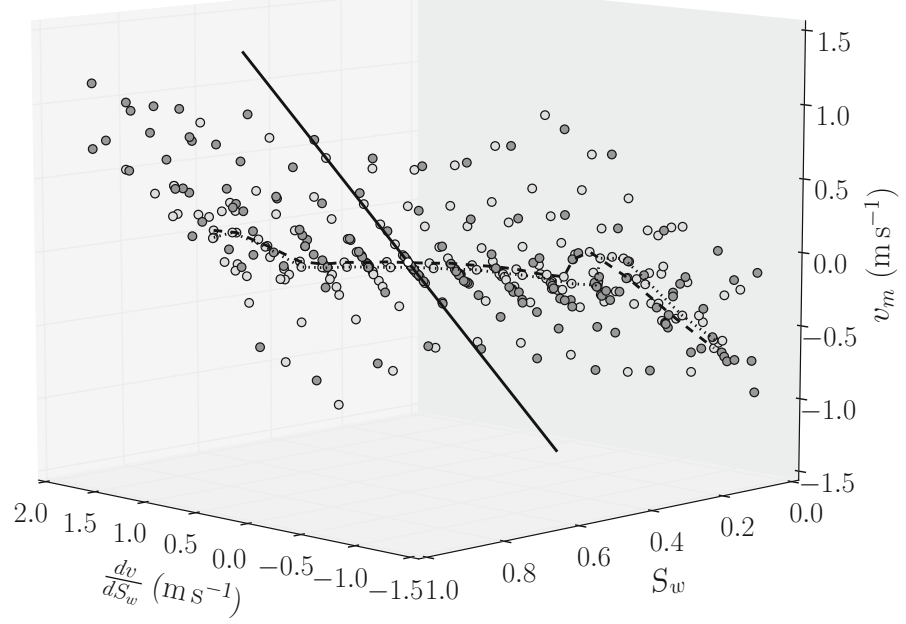

Fig. 5 Plot of $v_{m}$ as a function of $S_{w}$ and $\mathrm{d} v / \mathrm{d} S_{w}$ for a number of different flow parameters for both the hexagonal lattices (light gray data points) and the square lattices (dark gray data points). The point $\left(S_{w}, v_{m}, v^{\prime}\right)=(0.6,0,0)$ is highlighted as a white circle. The straight line is $0.79 v^{\prime}$, making $b=0.79$ in Eq. (90). The dotted and the dashed curves correspond to the two datasets shown in Fig. 7: The curve passing through the point $\left(S_{w}, v_{m}, v^{\prime}\right)=(0.6,0,0)$ corresponds to the data set used for Fig. $7 \mathrm{~b}$, whereas the other curve is the data set corresponding to that of Fig. $7 \mathrm{a}$

predicts $S_{w}^{C}=S_{w}^{B}=S_{w}^{A}$, which is exactly what we find numerically. This constitutes a non-trivial test of the theory: From an experimental point of view, this is the point where $v_{w}=v_{n}=v$ and $v$ has its minimum. These are all measurable quantities.

\section{Discussion and Conclusion}

We have here introduced a new thermodynamic formalism to describe immiscible two-phase flow in porous media. This work is to be seen as a first step toward a complete theory based on equilibrium and non-equilibrium thermodynamics. In fact, the only aspect of thermodynamic analysis that we have utilized here is the recognition that the central variables are Euler homogeneous functions. This has allowed us to derive a closed set of equations in Sect. 6 . This was achieved by introducing the co-moving velocity (47). This is a velocity function that relates the seepage velocities $v_{w}$ and $v_{n}$ to the thermodynamic velocities $\hat{v}_{w}$ and $\hat{v}_{n}$ defined through the Euler theorem in Sect. 3.

The theory we have developed here is "kinetic" in the sense that it only concerns relations between the velocities of the fluids in the porous medium and not relations between the driving forces that generate these velocities. We may use the term "dynamic" about these latter relations.

The theory rests on a description on two levels, or scales. The first one is the pore scale. Then, there is REV scale. This scale is much larger than the pore scale so that the porous medium is differentiable. At the same time, we assume the REV to be so small that we may assume that the flow through it is homogeneous and at steady state. At scales much larger than the REV scale, we do not assume steady-state flow. Rather, saturations and velocity fields may vary both in space and time. It is at this scale that the equations in Sect. 6 apply. 

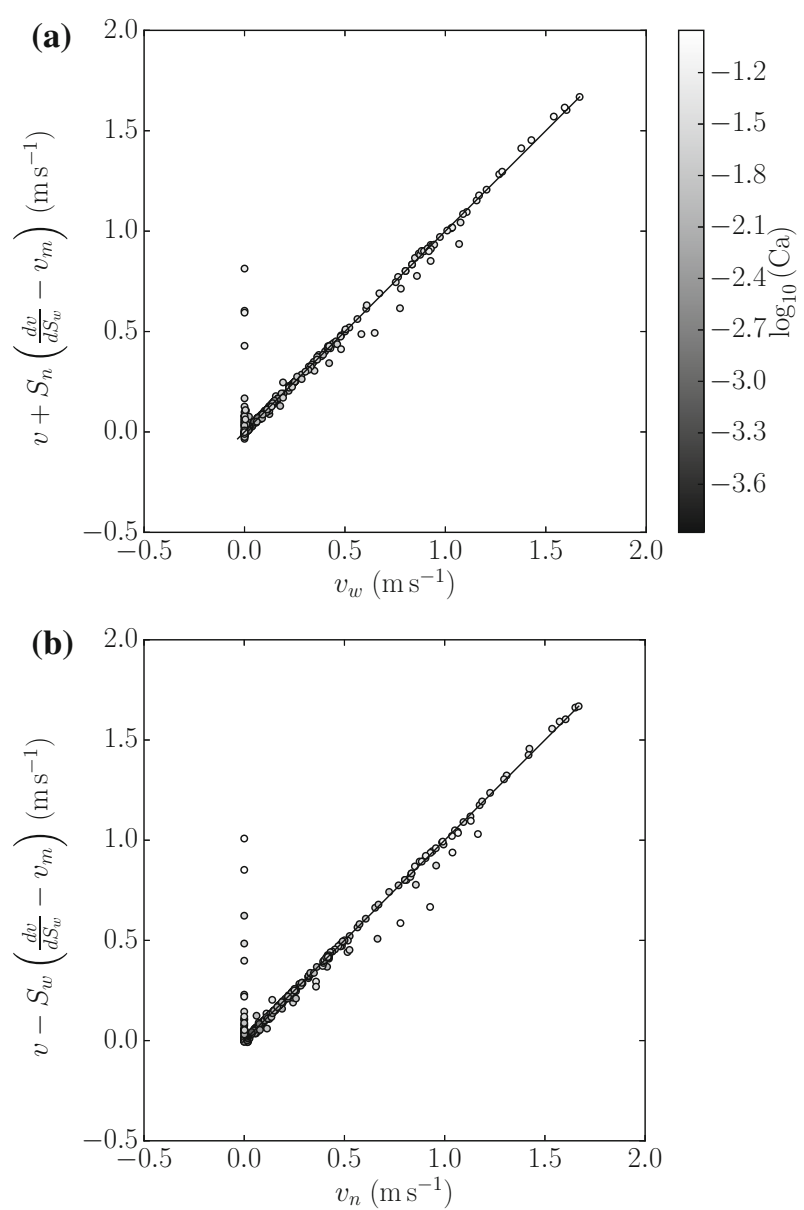

Fig. 6 We show the seepage velocities $v_{w}$ (a) and $v_{n}$ (b) calculated from Eqs. (42) and (43) versus the measured $v_{w}$ and $v_{n}$. The data points are shaded according to the capillary number $\mathrm{Ca}$ of the flow and are based on both the hexagonal and the square lattices

The necessity to introduce the co-moving velocity $v_{m}$ is surprising at first. However, it is a result of the homogenization procedure when going from the pore scale to the REV scale: The pore geometry imposes restrictions on the flow which must reflect itself at the REV scale.

One may at this point ask what has been gained in comparison with the relative permeability formalism? The relative permeability approach consists in making explicit assumptions about the functional form of $v_{w}$ and $v_{n}$ through the generalized Darcy equations Bear (1988). The relative permeability formalism thus reduces the immiscible two-phase problem to the knowledge of three functions $k_{r, w}=k_{r, w}\left(S_{w}\right), k_{r, n}=k_{r, w}\left(S_{n}\right)$ and $P_{c}=P_{c}\left(S_{w}\right)$. However, strong assumptions about the flow have been made in order to reduce the problem to these three functions: Assumptions that are known to be at best approximative.

Our approach leading to the central equations in Sect. 6 does not make any assumptions about the flow field beyond the scaling assumption in Eq. (15). Hence, the constitutive Eq. (48) can have any form, for example the nonlinear one presented in Tallakstad et al. (2009a, b), 

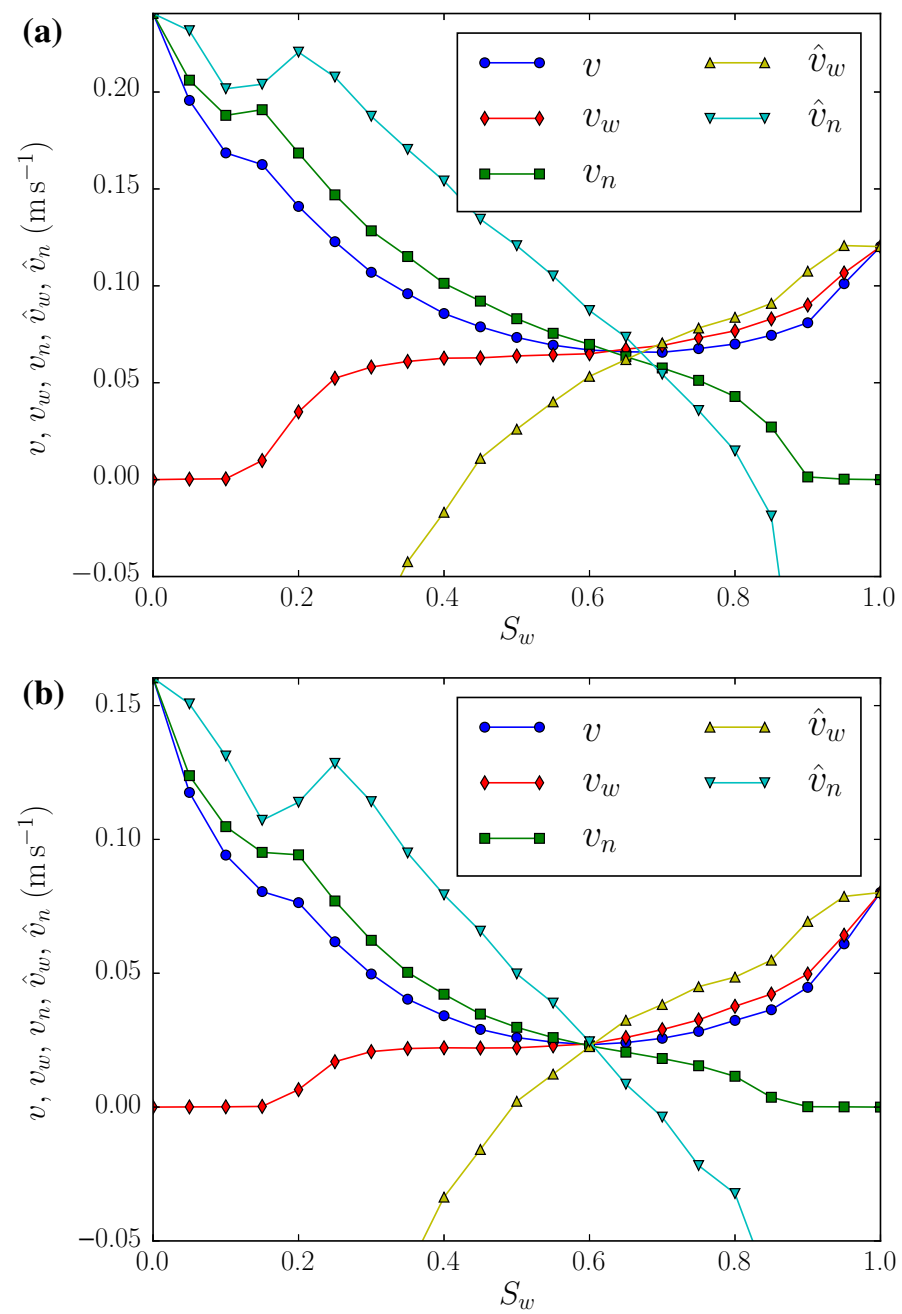

Fig. 7 Plot of $v, v_{w}, v_{n}, \hat{v}_{w}$ and $\hat{v}_{n}$ versu $S_{w}$ for the hexagonal lattice. In a, we have set $\Delta P / L=-0.15 \mathrm{MPa} / \mathrm{m}$, $\eta_{w}=0.001 \mathrm{Pas}, \eta_{n}=0.002 \mathrm{Pas}$ and $\sigma=0.03 \mathrm{~N} / \mathrm{m}$. The dataset corresponds to the dotted curve in Fig. 5 for which $v_{m}=0$ does not coincide with $v^{\prime}=0$. We see that the three cross points defined in Sect. 5 are ordered according to inequality (41), $S_{w}^{C} \leq S_{w}^{B} \leq S_{w}^{A}$. In $\mathbf{b}$, we have set $\Delta P / L=-0.10 \mathrm{MPa} / \mathrm{m}, \eta_{w}=0.001 \mathrm{Pas}$, $\eta_{n}=0.002 \mathrm{Pas}$ and $\sigma=0.03 \mathrm{~N} / \mathrm{m}$. The dataset corresponds to the dashed curve in Fig. 5 passing through $\left(v_{m}, v^{\prime}\right)=(0,0)$. In this case, we find that the cross points all coincide. Hence, we have $S_{w}^{C}=S_{w}^{B}=S_{w}^{A}$, as predicted

Rassi et al. (2011), Sinha and Hansen (2012) and Sinha et al. (2017). Hence, our approach is in this sense much more general than the relative permeability one.

Acknowledgements The authors thank Carl Fredrik Berg, Eirik Grude Flekkøy, Knut Jørgen Måløy, Thomas Ramstad, Isha Savani, Per Arne Slotte, Marios Valavanides and Mathias Winkler for interesting discussions on this topic. We also thank the anonymous reviewers for helpful comments. AH and SS thank the Beijing Computational Science Research Center (CSRC) for financial support and hospitality. SS was supported by National Natural Science Foundation of China under Grant Number 11750110430. This work was partly 
supported by the Research Council of Norway through its Centres of Excellence funding scheme, Project Number 262644.

Open Access This article is distributed under the terms of the Creative Commons Attribution 4.0 International License (http://creativecommons.org/licenses/by/4.0/), which permits unrestricted use, distribution, and reproduction in any medium, provided you give appropriate credit to the original author(s) and the source, provide a link to the Creative Commons license, and indicate if changes were made.

\section{References}

Aker, E., Måløy, K.J., Hansen, A., Batrouni, G.G.: A two-dimensional network simulator for two-phase flow in porous media. Transp. Porous Media 32, 163 (1998)

Bear, J.: Dynamics of Fluids in Porous Media. Dover, Mineola (1988)

Bentsen, R.G., Trivedi, J.: On the construction of an experimentally based set of equations to describe cocurrent or countercurrent, two-phase flow of immiscible fluids through porous media. Transp. Porous Media 99, 251 (2013)

Buckley, S.E., Leverett, M.C.: Mechanism of fluid displacements in sands. Trans. AIME 146, 107 (1942)

Döster, F., Hönig, O., Hilfer, R.: Horizontal flow and capillarity-driven redistribution in porous media. Phys. Rev. E 86, 016317 (2012)

Ghanbarian, B., Sahimi, M., Daigle, H.: Modeling relative permeability of water in soil: application of effectivemedium approximation and percolation theory. Water Resour. Res. 52, 5025 (2016)

Gray, W.G., Hassanizadeh, S.M.: Averaging theorems and averaged equations for transport of interface properties in multiphase systems. Int. J. Multiph. Flow 15, 81 (1989)

Gray, W.G., Miller, C.T.: Thermodynamically constrained averaging theory approach for modeling flow and transport phenomena in porous medium systems: 1. Motivation and overview. Adv. Water Res. 28, 161 (2005)

Gray, W.G., Miller, C.T.: Introduction to Thermodynamically Constrained Averaging Theory for Porous Medium Systems. Springer, Berlin (2014)

Hassanizadeh, S.M.: Advanced theories for two-phase flow in porous media. In: Vafai, K. (ed.) Handbook of Porous Media, 3rd edn. CRC Press, Boca Raton (2015)

Hassanizadeh, S.M., Gray, W.G.: Mechanics and thermodynamics of multiphase flow in porous media including interphase boundaries. Adv. Water Res. 13, 169 (1990)

Hassanizadeh, S.M., Gray, W.G.: Towards an improved description of the physics of two-phase flow. Adv. Water Res. 16, 53 (1993)

Hassanizadeh, S.M., Gray, W.G.: Thermodynamic basis of capillary pressure in porous media. Water Resour. Res. 29, 3389 (1993)

Hilfer, R.: Macroscopic equations of motion for two-phase flow in porous media. Phys. Rev. E 58, 2090 (1998)

Hilfer, R.: Capillary pressure, hysteresis and residual saturation in porous media. Physica A 359, 119 (2006a)

Hilfer, R.: Macroscopic capillarity and hysteresis for flow in porous media. Phys. Rev. E 73, 016307 (2006b)

Hilfer, R.: Macroscopic capillarity without a constitutive capillary pressure function. Physica A 371, 209 (2006c)

Hilfer, R., Besserer, H.: Macroscopic two-phase flow in porous media. Physica B 279, 125 (2000)

Hilfer, R., Döster, F.: Percolation as a basic concept for capillarity. Transp. Porous Media 82, 507 (2010)

Hilfer, R., Armstrong, R.T., Berg, S., Georgiadisand, A., Ott, H.: Capillary saturation and desaturation. Phys. Rev. E 92, 063023 (2015)

Kjelstrup, S., Bedeaux, D.: Non-equilibrium Thermodynamics for Heterogeneous Systems. World Scientific, Singapore (2008)

Kjelstrup, S., Bedeaux, D., Johannesen, E., Gross, J.: Non-equilibrium Thermodynamics for Engineers, 2nd edn. World Scientific, Singapore (2017)

Kondepudi, D., Prigogine, I.: Modern Thermodynamics. Wiley, Chichester (1998)

Larson, R.G., Scriven, L.E., Davis, H.T.: Percolation theory of two phase flow in porous media. Chem. Eng. Sci. 36, 57 (1981)

Leverett, M.C.: Capillary behavior in porous sands. Trans. AIMME 12, 152 (1940)

Niessner, J., Berg, S., Hassanizadeh, S.M.: Comparison of two-phase Darcy's law with a thermodynamically consistent approach. Transp. Porous Media 88, 133 (2011)

Rassi, E.M., Codd, S.L., Seymour, J.D.: Nuclear magnetic resonance characterization of the stationary dynamics of partially saturated media during steady-state infiltration flow. N. J. Phys. 13, 015007 (2011)

Richards, L.A.: Capillary conduction of liquids through porous mediums. J. Appl. Phys. 1, 318 (1931) 
Savani, I., Bedeaux, D., Kjelstrup, S., Sinha, S., Vassvik, M., Hansen, A.: Ensemble distribution for immiscible two-phase flow in porous media. Phys. Rev. E. 95, 023116 (2017a)

Savani, I., Sinha, S., Hansen, A., Bedeaux, D., Kjelstrup, S., Vassvik, M.: A Monte Carlo algorithm for immiscible two-phase flow in porous media. Transp. Porous Media 116, 869 (2017b)

Scheidegger, A.E.: Theoretical models of porous matter. Prod. Mon. 17, 17 (1953)

Scheidegger, A.E.: The Physics of Flow Through Porous Media. University of Toronto Press, Toronto (1974)

Sinha, S., Gjennestad, M.A., Vassvik, M., Winkler, M., Hansen, A., Flekkøy, E.G.: Rheology of high-capillary number flow in porous media. in preparation (2018)

Sinha, S., Hansen, A.: Effective rheology of immiscible two-phase flow in porous media. Europhys. Lett. 99, 44004 (2012)

Sinha, S., Hansen, A., Bedeaux, D., Kjelstrup, S.: Effective rheology of bubbles moving in a capillary tube. Phys. Rev. E 87, 025001 (2013)

Sinha, S., Bender, A.T., Danczyk, M., Keepseagle, K., Prather, C.A., Bray, J.M., Thrane, L.W., Seymor, J.D., Codd, S.L., Hansen, A.: Effective rheology of two-phase flow in three-dimensional porous media: experiment and simulation. Trans. Porous Media 119, 77 (2017)

Tallakstad, K.T., Knudsen, H.A., Ramstad, T., Løvoll, G., Måløy, K.J., Toussaint, R., Flekkøy, E.G.: Steadystate two-phase flow in porous media: statistics and transport properties. Phys. Rev. Lett. 102, 074502 (2009a)

Tallakstad, K.T., Løvoll, G., Knudsen, H.A., Ramstad, T., Flekkøy, E.G., Måløy, K.J.: Steady-state simultaneous two-phase flow in porous media: an experimental study. Phys. Rev. E 80, 036308 (2009b)

Wyckoff, R.D., Botset, H.G.: The flow of gas-liquid mixtures through unconsolidated sands. J. Appl. Phys. 7, 325 (1936) 